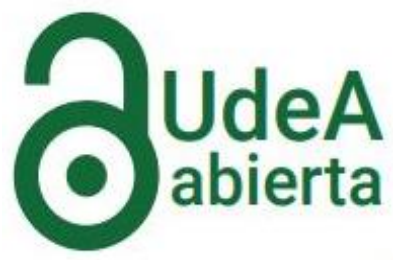

I I B I C R I T

CONICET

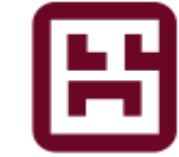

CEDES

Centro de Estudios

de Estado y Sociedad
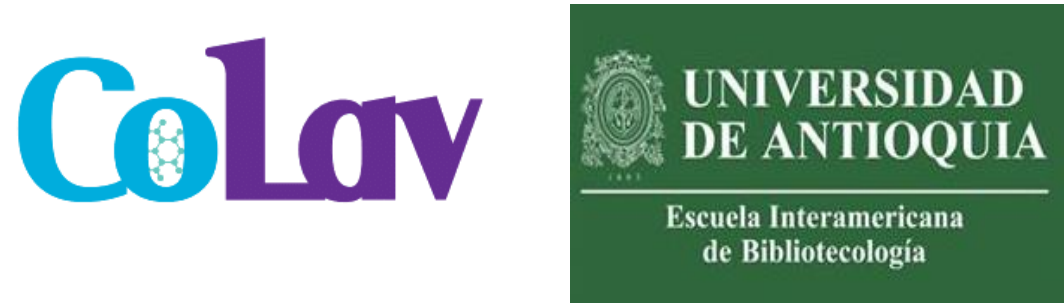

$\overline{\mathrm{HUMA}} \overline{\mathrm{NIDAD}} \overline{\mathrm{ES}}$

DIGITALES
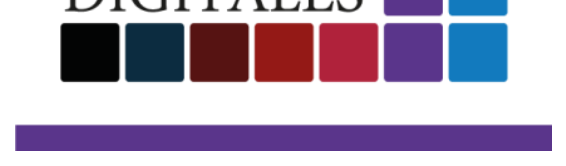

CONICET

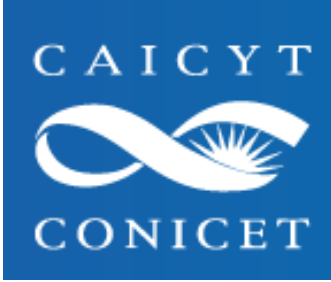

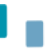

10

1.

- Hospital El Cruce

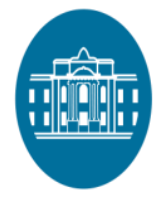

SISTEMA DE GESTIÓN

DEL CONOCIMIENTO

ANLIS MALBRÁN

\title{
Open Science since Covid-19: Open Access + Open Data
}

Alejandro Uribe-Tirado - Universidad de Antioquia. CoLaV. EIB (Colombia) auribe.bibliotecologia.udea@gmail.com - alejandro.uribe2@udea.edu.co

Gimena del Rio Riande - IIBICRIT.CONICET; HUMANIDADES DIGITALES; CAICYT (Argentina) gdelrio.riande@gmail.com

Sandra Raiher - CEDES; Hospital EI Cruce; SGC ANLIS Malbrán (Argentina) sandraraiher@gmail.com

Jaider Ochoa-Gutiérrez - Universidad de Antioquia. CoLaV. EIB (Colombia) jaider.ochoa@udea.edu.co 
The coronavirus crisis has created different initiatives that promote access to open publications and open data, solve collaboratively and from different places, being an example of the benefits of open science. From the initial version of the Compilation on Open Science from COVID-19: Open Access + Open Data (Version I: April 3, 2020) published by Alejandro Uribe-Tirado (http://eprints.rclis.org/39864/), it seemed to us, a good practice to update this first input openly and collaboratively, using the platform: https://etherpad.wikimedia.org/p/covid19. This new version (Version II: June 3, 2020), is the result of this joint work.

\section{Analysis}

- CoLaV / COVID-19 (Most significant production among the different studies around the world on COVID-19)

https://onodo.org/visualizations/115523 (Beta)

\section{- Critical Analysis and Systematization}

- OCDE-OECD: Tackling coronavirus - Why open science is critical to combatting COVID-19:

https://www.oecd.org/coronavirus/en/?utm source=Adestra\&utm medium=email\&utm content=Ta ckling\%20coronavirus\%3A\%20contributing\%20to\%20a\%20global\%20effort\&utm campaign=OEC D\%20Science\%2C\%20Technology\%20\%26\%20Innovation\%20News\%2005\%2F2020\&utm term= demo

- Pensar la pandemia. Observatorio Social del Coronavirus (CLACSO): https://www.clacso.org/pensar-la-pandemiaobservatorio-m-del-coronavirus/

- CEPAL-COVID-19: https://www.cepal.org/es/publicaciones/45337-america-latina-caribe-lapandemia-covid-19-efectos-economicossociales?utm source=CiviCRM\&utm medium=email\&utm campaign=20200407 informe covid19

- Respuestas de los sistemas educativos de América Latina a la crisis de la COVID-19: https://www.siteal.iiep.unesco.org/respuestas educativas covid 19?utm source=email marketing \&utm admin=132116\&utm medium=email\&utm campaign=Cmo responden los sistemas educa tivos de la regin a la COVID Lea la sistematizacin en el SITEAL

- Educación y pandemia. Una visión académica: https://www.iisue.unam.mx/investigacion/textos/educacion pandemia.pdf

- Coronavirus SARS-CoV-2 y patentes: https://abg-ip.com/es/coronavirus-patentes/?cli action=1587573221.233

\section{- Associations (Agreements to facilitate access to information and publications)}

- American Association for the Advancement of Science. Latest news and research on Covid-19.: https://www.sciencemag.org/tags/coronavirus

- American Academy of Pediatrics.Critical Updates and resources for pediatricians: https://services.aap.org/en/pages/2019-novel-coronavirus-covid-19-infections/

- American College of Physicians. A collection of Annals of Internal Medicine articles related to coronavirus is freely available:

https://annals.org/aim/pages/coronavirus-content

- American Institute of Physics. AIP Publishing has made a collection of articles: https://publishing.aip.org/publications/journals/covid-19/

- American Medical Association. JAMA Network COVID-19 information collection: https://jamanetwork.com/journals/jama/pages/coronavirus-alert

- American Psychiatric Association Publishing. Resources for Psychiatrists and on Coping with the Outbreak: https://www.psychiatry.org/news-room/apa-blogs/apa-blog/2020/03/covid-19-mental-healthimpacts-resources-for-psychiatrists 
- American Physiological Society. Journal articles, resources, news articles and other information to keep you informed and help you work smarter and more efficiently from home: https://www.physiology.org/career/teaching-learning-resources/covid-19-resources?SSO=Y

- American Society of Civil Engineers. Resources for working from home: https://collaborate.asce.org/covid-19/home

- American Society of Clinical Oncology. General resources and information: https://www.asco.org/asco-coronavirus-information

- American Society of Nephrology. Resources for Outpatient Dialysis Facilities: https://www.asn-online.org/ntds/

- American Society for Microbiology. Free access to research articles about COVID-19 \& toolkit to help spread information: https://www.asm.org/Press-Releases/2020/COVID-19-Resources

- Asociación Regional de Diálisis y Trasplantes Renales (Argentina): Recursos de información especializados sobre COVID-19 SARS-CoV-2 en relación con la práctica nefrológica:

https://sites.google.com/view/covid-19-nefrologa/home

- China National Knowledge Infrastructure. IE Library comparte la plataforma CNKI, con los logros de investigación en China y el mundo.

http://en.gzbd.cnki.net/GZBT/brief/Default.aspx

- European Respiratory Society. The latest articles from ERS publications on SARS-CoV-2 and COVID-19:

https://erj.ersjournals.com/cc/sars-cov-2-and-covid-19

- Future Science Group - Infectious Diseases Hub. Discover the latest updates on the ongoing novel coronavirus outbreak in the 'COVID-19 Hub'

https://www.id-hub.com/2020/03/17/in-focus-coronavirus/

- Microbiology Society. Microbiology Society has brought together articles from across their portfolio and made this content freely available:

https://www.microbiologyresearch.org/content/coronaviruses

- Royal Society of Chemistry. Coronavirus articles - free to access collection: https://pubs.rsc.org/en/Journals/ArticleCollectionLanding?themeld=1eac56a1-4121-43c5-b3ec3d6f9d9226fb\&journalName

- The Royal Society. Sharing research data and findings relevant to the novel coronavirus outbreak: https://royalsocietypublishing.org/topic/special-collections/novel-coronavirus-outbreak

\section{- Knowledge base on epidemics and pandemics}

- AmeliCA: http://amelica.org/epidemics/ (SPARQL endpoint)

- CLACSO: https://www.clacso.org/base-de-conocimiento-en-epidemias-pandemias-de-revistas/

\section{- Databases and Repositories with open content}

Multidisciplinary open science platforms

- BioRxiv and MedRxiv:

https://connect.biorxiv.org/relate/content/181 - https://connect.medrxiv.org/relate/content/181

- Dimensions:

https://covid-19.dimensions.ai/

- Figshare:

https://covid19.figshare.com/

- Frontiers Coronavirus Knowledge Hub:

https://coronavirus.frontiersin.org/?utm source=ad\&utm medium=tw\&utm campaign

- KUDOS Pro:

https://growkudos.com/projects/covid-19 
Science Open. Novel Coronavirus Disease COVID-19:

https://www.scienceopen.com/collection/COVID 19

- ZENODO - Open AIRE Community - Gateway:

https://www.openaire.eu/openaire-activities-for-covid-19

\section{Others}

- Base:

https://phgkb.cdc.gov/PHGKB/coVInfoStartPage.action

- ClinicalTrials:

https://clinicaltrials.gov/ct2/results?cond=covid19+OR+covid-

19+OR+\%22covid+19\%22+OR+2019ncov+OR+2019-

ncov+OR+\%222019+ncov\%22+OR+\%222019+Novel+Coronavirus\%22+OR+\%222019+novel+cov $\% 22+O R+\% 28$ wuhan+AND+\%28ncov+OR+coronavirus \%29\%29\&term=\&cntry=\&state=\&city=\&dis $\mathrm{t}=\&$ Search $=$ Search

- Coronavirus COVID-19. National Institutes for Health USA:

https://www.nih.gov/health-information/coronavirus

- DisasterLit:

https://disasterinfo.nlm.nih.gov/search/?q=\%22covid-

19\%22\%200R\%20(covid\%20AND\%2019)\%20OR\%20(coronavirus\%20AND\%20novel)\%200R\%2

oWuhan\%200R\%20ncov

- GenBank COVID-2019 Sequence:

o https://www.ncbi.nlm.nih.gov/genbank/2019-ncov-

seqs/?utm campaign=wuhan nCoV\&utm source=insights\&utm medium=referral

- Europe PMC:

https://europepmc.org/search?query=covid $19 \% 200 R \% 20$ covid-

19\%200R\%20\%22covid\%2019\%22\%200R\%202019ncov\%200R\%202019-

ncov\%200R\%20\%222019\%20ncov\%22\%200R\%20\%222019\%20Novel\%20Coronavirus\%22\%20 OR\%20\%222019\%20novel\%20cov\%22\%200R\%20\%28wuhan\%20AND\%20\%28ncov\%200R\%2

0coronavirus\%29\%29\%200R\%20\%22novel\%20coronavirus\%20pneumonia\%22

- LitCovid:

https://www.ncbi.nlm.nih.gov/research/coronavirus/

- NCBI Virus' COVID-2019 data hub:

https://www.ncbi.nlm.nih.gov/labs/virus/vssi/\#/virus?SeqType s=Nucleotide\&VirusLineage ss=Wu han\%20seafood\%20market\%20pneumonia\%20virus,\%20taxid:2697049

- Public Health Genomics and Precision Health Knowledge:

https://phgkb.colc.gov/PHGKB/phgHome.action?action=home

- PUBLONS:

https://publons.com/publon/covid-19/?sort by=date

- PubMed:

https://www.ncbi.nlm.nih.gov/pubmed/?term=\%22Wuhan+coronavirus $\% 22 \% 5 B S u p p l e m e n t a r y+C o$ ncept $\% 5$ D+OR+covid 19+OR+covid-19+OR+\%22covid+19\%22+OR+2019ncov+OR+2019-

ncov+OR+\%222019+ncov\%22+OR+\%222019+Novel+Coronavirus\%22+OR+\%222019+novel+cov $\% 22+\mathrm{OR}+($ wuhan+AND+(ncov+OR+coronavirus))+OR+\%22novel+coronavirus+pneumonia\%22

- WHO COVID-19 research article database:

https://www.who.int/emergencies/diseases/novel-coronavirus-2019/global-research-on-novel-

coronavirus-2019-ncov

\section{Others. Latin America}

- BIREME/OPS/OMS. Enfermedad por coronavirus:

https://bvsalud.org/vitrinas/es/post vitrines/nuevo coronavirus/

- CLACAI. Colección COVID-19:

https://clacaidigital.info/handle/123456789/1284 
- Observatorio OEI (Seguimiento en tiempo real desde datos de Pubmed):

https://observatoriocts.oei.org.ar/2020/03/25/el-radar-del-observatoriocts-seguimiento-en-vivo-delcovid-19/

- OPS. Repositorio Orientación sobre la COVID-19 y últimas investigaciones en las Américas:

https://covid19-evidence.paho.org/

- Repositorio Digital de la Universidad Juan Agustín Maza. Colección COVID19:

http://repositorio.umaza.edu.ar/handle/00261/1050

- Sistema Nacional de Repositorios Digitales de Argentina. Colección COVID-19:

https://repositoriosdigitales.mincyt.gob.ar/vufind/Search/Results?lookfor=\%22COVID19\%22+OR+\%222019-nCoV\%22+OR+\%E2\%80\%9CSARS-CoV-2\%E2\%80\%9D\&type=AllFields

- Search and production. (According to purpose and specialization)

- ALIA: https://www.alia.org.au/groups/HLA/covid-19-live-literature-searches

- CCSS: https://sites.google.com/view/covid19-por-especialidades/p\%C3\%A1ginaprincipal?authuser $=0$

- ECRI: https://www.ecri.org/coronavirus-covid-19-outbreak-preparedness-center

- Communities (Social Networks)

- Open Source COVID19 Medical Supplies: https://www.facebook.com/groups/670932227050506/

- Dataset on Covid-19 (Open data)

- CAS:

https://www.cas.org/covid-19-antiviral-compounds-dataset

- COVID-19 Open Research Dataset:

https://pages.semanticscholar.org/coronavirus-research

- Datasets Search - Google:

https://datasetsearch.research.google.com/search?query=coronavirus $\% 20$ covid-

19\&docid=g3oiDuHtkLygNkWHAAAAAA\%3D\%3D

- GenBank COVID-19 Sequences:

https://www.ncbi.nlm.nih.gov/genbank/2019-ncov-

segs/?utm campaign=wuhan nCoV\&utm source=insights\&utm medium=referral

- LENS:

https://about.lens.org/covid-19/

- NCBI Virus' COVID-19 data hub:

https://www.ncbi.nlm.nih.gov/labs/virus/vssi/\#/virus?SeqType s=Nucleotide\&VirusLineage ss=Wu han\%20seafood\%20market\%20pneumonia\%20virus,\%20taxid:2697049

- Open ICPSR:

https://www.openicpsr.org/openicpsr/search/covid19/studies

- Data and visualizations in real time (Dashboards)

\section{Principal}

- World Health Organization's online dashboard: http://who.maps.arcgis.com/apps/opsdashboard/index.htm|\#/c88e37cfc43b4ed3baf977d77e4a0667

- Johns Hopkins' online dashboard of reported cases:

- https://bit.ly/3d3o1km - https://coronavirus.jhu.edu/map.html 


\section{Other}

- Baidu online dashboard:

https://voice.baidu.com/act/newpneumonia/newpneumonia/?from=osari pc 3

- Early Alert's online dashboard:

https://early-

alert.maps.arcgis.com/apps/opsdashboard/index.htm|\#/20bfbf89c8e74c0494c90b1ae0fa7b78

- FAA online dashboard:

https://c3eon.maps.arcgis.com/apps/opsdashboard/index.html\#/ba9350bd7ee94db896e15640a1b 524c7

- STATISTA:

https://www.statista.com/page/covid-19-coronavirus

- Track Corona Live:

https://www.trackcorona.live/

- Declarations on open access to publications and data, due to the Covid-19 crisis

- Coalición Internacional de Consorcios de Bibliotecas-ICOLC: https://drive.google.com/file/d/1csWSS6IUHTAFdyVu100jisOo2cR3mLIB/view

- CODATA, GO FAIR, RDA and WDS:

https://codata.org/wp-content/uploads/2020/03/Data-Together-COVID-19-Statement-FINAL.pdf

- REBIUN:

https://www.rebiun.org/sites/default/files/2020-

03/Declaracionafavordelconocimientoabiertoysostenible.pdf

- Search strategies according to information needs and disciplines

- DIIGO:

https://www.diigo.com/profile/covid-19?query=\%23B\%C3\%BAsquedas bibliogr\%C3\%A1ficas

- BV Salud BIREME/OPS/OMS:

https://bvsalud.org/queries/?s=\%\&l=es ES

- Official sources to consult about COVID-19 in different countries

- Ibero-America:

https://fundaciongabo.org/es/noticias/articulo/fuentes-oficiales-para-consultar-informacion-sobrecovid-19-en-paises-de

- Argentina. Ministerio de Salud de Argentina - Casa Rosada:

https://www.argentina.gob.ar/salud/coronavirus-COVID-19 - https://www.casarosada.gob.ar/

- Chile. Ministerio de Salud de Chile: https://www.minsal.cl/nuevo-coronavirus-2019-ncov/

- Colombia. Ministerio de Salud y Protección Social de Colombia: http://186.31.104.166/ https://d2jsqrio60m94k.cloudfront

- Costa Rica. Ministerio de Salud de Costa Rica: https://www.ministeriodesalud.go.cr/

- Cuba. Ministerio de Salud Pública de la República de Cuba: https://salud.msp.gob.cu/

- Ecuador. Ministerio de Salud Pública de Ecuador: https://www.salud.gob.ec/coronavirus-covid$\underline{19 /}$

- Spain. Ministerio de Sanidad, Consumo y Bienestar Social: https://www.mscbs.gob.es/

- Guatemala. Ministerio de Salud Pública y Asistencia Social: https://www.mspas.gob.gt/ 
- Honduras. Secretaría de Salud de Honduras:

http://www.salud.gob.hn/site/index.php/secretiaria

- Mexico. Secretaría de Salud de México: https://www.gob.mx/salud

- Paraguay. Ministerio de Salud de Paraguay: https://www.mspbs.gov.py/index.php

- Peru. Ministerio de Salud del Perú: https://www.gob.pe/minsal

- Portugal. Servicio Nacional de Saúde. https://covid19.min-saude.pt/

- Dominican Republic. Ministerio de Salud Pública de República Dominicana: https://www.facebook.com/SaludPublicaRD/

- Venezuela. Ministerio del Poder Popular para la Salud: http://www.mpps.gob.ve/

- Uruguay. Ministerio de Salud Pública: https://www.gub.uy/ministerio-salud-publica/

Other countries:

- Germany: Taggeschau.de. https://www.tagesschau.de/inland/coronavirus-karte-deutschland101.html - Berliner MorgenPost Mapa https://interaktiv.morgenpost.de/corona-virus-karteinfektionen-deutschland-weltweit/

- Bélgica. SPF Santé publique, Sécurité de la Chaîne alimentaire et Environnement Comunicados prensa. https://www.infocoronavirus.be/fr/news/

- France. Suivi des Pacientes Covid-19 en Francia. https://mapthenews.maps.arcgis.com/apps/opsdashboard/index.html\#/5df19abcf8714bc590a3b 143e14a548c - Eficiens. https://www.eficiens.com/coronavirus-statistiques/ - Santé Publique France. https://www.santepubliquefrance.fr/maladies-et-traumatismes/maladies-etinfectionsrespiratoires/infection-a-coronavirus/articles/infection-au-nouveau-coronavirus-sarscov-2-covid-19-france- et-monde

- Irlanda: RTE COVID-19 National summary based on data from the Department of Health. https://www.rte.ie/news/coronavirus/summary/

- Italia. Ministero della Salute. http://www.salute.gov.it/nuovocoronavirus

- Switzerland. Swissinfo.ch. https://www.swissinfo.ch/spa/sociedad/coronavirus--lasituaci\%C3\%B3n-en-suiza/45592694

- Netherland. Ministerie van Volksgezondheid, Welzikn en Sport. https:/www.rivm.nl/actueleinformatie-over-coronavirus

- United Kingdom: BBC. https://www.bbc.com/news/uk-51768274 - Public Health Englandhttps://www.arcgis.com/apps/opsdashboard/index.html\#/f94c3c90da5b4e9f9a0b19484dd4bb14 Gov.UK https://www.gov.uk/government/news/nhs-asks-people-to-share-their-coronavirussymptoms-to-help-others

\section{- Protocols based on scientific evidence}

- Biblioteca Cochrane (Collection series on COVID-19. Available in: Simplified Chinese, German, Persian, French, Japanese, Malay, Polish, Portuguese, Russian, and English).

- Evidencia relevante para cuidados críticos: https://www.cochranelibrary.com/es/collections/doi/SC000039/full?contentLanguage=es

- Medidas de prevención y control de la infección: https://www.cochranelibrary.com/es/collections/doi/SC000040/full

\section{- Collaborative Research Initiatives}

- An initiative from the scientific community to put all available resources.COVID-19 https://crowdfightcovid19.org/

- Digital Narratives of COVID-19: https://github.com/dh-miami/narratives covid19 yhttps://covid.dh.miami.edu/ 
- Support initiatives about open access, open data and training on COVID-19 from Libraries

- ALA-ACRL:

https://acrl.libguides.com/pandemic/

- ICOLC:

https://icolc.net/statement/statement-global-covid-19-pandemic-and-its-impact-library-services-andresources

- IFLA:

https://www.ifla.org/ES/node/92983

- ITHAKA:

https://sr.ithaka.org/our-work/covid-19/

- General Information

- Wikipedia, Coronavirus:

https://en.wikipedia.org/wiki/Coronavirus

- Ibero-american interactive maps on COVID-19

- OEl: https://www.oei.es/covid-19-ciencia/investigacion

- Media

- New York Times, Coronavirus Map: Tracking the Global Outbreak:

https://www.nytimes.com/interactive/2020/world/coronavirus-maps.html

- New York Time, Coronavirus News:

https://www.nytimes.com/news-event/coronavirus

- Metasearch - Search Engine

- COVID Scholar

https://www.covidscholar.org/

- C3.ai COVID-19 Data Lake

https://c3.ai/products/c3-ai-covid-19-data-lake/

- INFO COVID-19 (en español o en inglés)

https://cse.google.com/cse?cx=017334360823949002299:gyqmwoprjwp

- Multimedia

- Primal Pictures. (Data and findings relevant to the novel coronavirus / COVID-19

disease):https://www.primalpictures.com/disease-and-conditions-covid-19/

\section{- Verified news}

- Corona virus disease. Curated news and resources on COVID-19 (Curated by the Scoop.it team): https://www.scoop.it/topic/corona-virus-news 
- Fake news (Information and Training to avoid them)

- Spain:

https://maldita.es/coronavirus/

- Colombia:

https://colombiacheck.com/especiales/coronavirus/

- Latin-America:

https://www.chequeado.com/latamcoronavirus/

- International:

https://www.factcheck.org/a-guide-to-our-coronavirus-coverage/

- International:

https://www.poynter.org/ifcn-covid-19-misinformation/

\section{- Patents}

- Patentscope Covid-19 Index:

https://patentscope.wipo.int/search/en/covid19.jsf

- Compilation of publications from reference management software

- Zotero:

https://www.zotero.org/groups/2475685/digital narratives of covid-19

- Mendeley

https://data.mendeley.com/collections/f082ece9-8143-4e19-8611-31f9973a0a2d

- Additional resources prepared by librarians or documentalists

- Blog de María García-Puente (@bibliovirtual):

https://bibliovirtual.wordpress.com/novedades-y-recursos/

- Networks on COVID-19 and open science (open data)

- Virus Outbreak Data Network (VODAN):

https://www.go-fair.org/implementation-networks/overview/vodan/

- Retractions

- Retration Wash. COVID-19:

https://retractionwatch.com/?s=COVID-19

- Journals and Publishers Databases (with Open Access Publications or open due to the crisis)

- American Society for Microbiology:

https://www.asm.org/Press-Releases/2020/COVID-19-Resources

- Annual Reviews:

https://annualreviewsnews.org/2020/03/13/annual-reviews-removes-access-control-in-response-tocovid-19-pandemic/ 
- BioOne (Avian Diseases, Journal of Wildlife Diseases, Journal of Zoo and Wildlife Medicine): https://complete.bioone.org/covid-19

- BMJ:

https://www.bmi.com/coronavirus?int source=wisepops\&int medium=wisepops\&int campaign=DA A CoronaVirus Jan24

- British Medical Journal: https://www.bmj.com/coronavirus

- Cambridge Core-Collection: https://www.cambridge.org/core/browse-subjects/medicine/coronavirus-free-access-collection

- China CDC Weekly: http://weekly.chinacdc.cn/

- Clarivate COVID-19: https://clarivate.com/coronavirus-resources/

- Copyright Clearance Center List: https://www.copyright.com/coronavirus-covid-19/

- COVID-TECA (Hemeroteca de humanidades sobre la pandemia de coronavirus): https://iberlab.ugr.es/hemeroteca-covid19/

- El Profesional de la Información: http://www.elprofesionaldelainformacion.com/contenidos/2020/may/index.html

- Emerald Publishing: https://www.emeraldgrouppublishing.com/promo/coronavirus.htm?utm source=smc email awaren ess\&utm medium=email\&utm campaign=pim Covid-

19\%20Announcement\%20S\&EE 20200330 free

- Eurosurveillance. COVID-19 Collection: https://www.eurosurveillance.org/content/2019-ncov

- F1000Research: https://f1000research.com/gateways/disease outbreaks/coronavirus

- Frontiers. Coronavirus Funding Monitor (curated list): https://coronavirus.frontiersin.org/covid-19-research-funding-monitor

- Gale Health and Wellness: https://go.gale.com/ps/start.do?p=HWRC\&u=geb trial

- Gideon (Global Infectious Diseases and Epidemiology Online Network): http://tools.ovid.com/coronavirus/\#gideonVideo

- Journal of the American Medical Association. COVID-19: collection: https://jamanetwork.com/journals/jama/pages/coronavirus-alertexternal

- Journal of Visualized Experiments (JoVE). COVID-19: collection: https://info2.jove.com/coronavirusvideoexternal

- JSTOR:

https://daily.jstor.org/teaching-pandemics/ - https://about.jstor.org/covid19/

- Lancet: COVID-19 collection: https://www.thelancet.com/coronavirusexternal

- MIT Press: https://mitpress.mit.edu/blog/articles-understanding-pandemics-and-epidemiology

- Nature:

http://sfx.library.cdc.gov/cdc?url ver=Z39.88-

2004\&url ctx fmt=info:ofi/fmt:kev:mtx:ctx\&ctx enc=info:ofi/enc:UTF-8\&ctx ver=Z39.882004\&rfr id=info:sid/sfxit.com:azlist\&sfx.ignore date threshold=1\&rft.object id=954925427238\&rft.obje ct portfolio id=110974981369255\&svc.fulltext=yes\&sfx.directlink=force

- New England Journal of Medicine: https://www.nejm.org/coronavirus 
- Proceedings of the National Academy of Sciences:

https://www.pnas.org/coronavirus

- Proquest:

https://www.proquest.com/products-services/related/Coronavirus-Outbreak-Authoritative-

Resources.html

- Sage. Coronavirus (COVID-19) Research:

https://journals.sagepub.com/coronavirus?utm source=Adestra\&utm medium=email\&utm content =0A00927\&utm campaign=not+tracked\&utm term=\&em=cdfe678a4c8264350f0d0313b06045b98 ccc2587ad78780fbcaf48505c1e8926

- Springer Nature:

https://www.springernature.com/gp/librarians/landing/covid19-library-resources?sap-outbound$\mathrm{id}=$ AEE162CE2226967739A62FA5B4A90B818AF77DEA\&utm source=hybris-

campaign\&utm medium=email\&utm campaign=106 BASC02 0000020398 Library\%20Link\%20NL\% 20Special\%20Issue\%20COVID\%2019\&utm content=EN internal 45377 20200402\&mktkey=005056B0331B1ED782E9179F94E28613

- Science:

https://science.sciencemag.org/

- Taylor \&Francis:

https://taylorandfrancis.com/coronavirus/

- The New England Journal of Medicine. COVID-19 collection: https://www.neim.org/coronavirusexternal

o Thomson Reuters: https://www.thomsonreuters.com/en/resources/covid-19.html

- Willey Online Library:

https://novel-coronavirus.onlinelibrary.wiley.com/

\section{- Collaborative and research works, multi-institutional and multi-country partnerships}

- Clinical Trials:

https://www.clinicaltrials.gov/ct2/show/NCT04321616?titles=SOLIDARITY\&draw=2

- DNDIAL:

https://www.dndial.org/es/2020/comunicacion-e-informacion/es-press-releases/se-lanza-unacoalicion-para-acelerar-las-investigaciones-sobre-prevencion-y-tratamiento-de-covid-19-en-paisesde-medianos-y-bajos-ingresos-2/

- RD-Alliance:

https://www.rd-alliance.org/groups/rda-covid19

\section{- Learning / teaching resources}

- A Snapshot of Higher Education's Response to the COVID-19 Pandemic:

https://docs.google.com/document/d/1JG5rJmOOTwNhPKWPmBZLXtnU5trS4X8ZyABJRLGNgzM ledit?fbclid=IwAR2TauoJW-odH40QkdNAzSX5QBhhtKYo-i0OtZES6hOxdXL0-aV-3i5r480

- Covid-19. The good news map:

https://covid.mehdibaha.com/en

- How professors changed their teaching in this spring's shift to remote learning | Inside Higher Ed: https://www.insidehighered.com/digital-learning/article/2020/04/22/how-professors-changed-theirteaching-springs-shift-remote?utm source=Inside+Higher+Ed\&utm campaign=0230b331 dfWNU COPY 01\&utm medium=email\&utm term=0 1fcbc04421-0230b331df$\underline{236389898 \& m c}$ cid=0230b331df\&mc eid=1ad0d983d8 
- Infographics:

https://embed.widencdn.net/pdf/plus/cengage/1yhakzkaya/higher-educations-response-to-covid19infographic-1369680.pdf/?utm campaign=in movetoonline dg fy2021\&\&utm content=1373605

- Survey: http://onlinelearningsurvey.com/covid.html

- The Covid Tracking Project: https://covidtracking.com/

- The News Beyond The COVID Numbers: https://www.npr.org/2020/04/21/840609912/the-news-beyond-the-covid-numbers

\section{- $\underline{\text { Twitter and COVID-19 }}$}

- A Twitter Dataset. 150+ million tweets related to COVID-19 for open research (Version 4.0): http://doi.org/10.5281/zenodo.3738018 - https://github.com/thepanacealab/covid19 twitter

- COVID-19: The First Public Coronavirus Twitter Dataset: https://arxiv.org/abs/2003.07372 - https://github.com/echen102/COVID-19-TweetIDs

- Coronavirus Tweet Ids.Harvard Dataverse, V2.: https://doi.org/10.7910/DVN/LWOBTB

- Corona Virus (COVID-19). Tweets Dataset. IEEE Dataport, 2020 http://dx.doi.org/10.21227/781w-ef42 - https://ieee-dataport.org/open-access/corona-virus-covid19-tweets-dataset

- Documenting the Now updated Catalogue: https://catalog.docnow.io/

- How did Twitter react to the coronavirus pandemic: https://towardsdatascience.com/how-did-twitter-react-to-the-coronavirus-pandemic-2857592b449a 